\title{
Anterior staircase manoeuvre for atrial transseptal puncture
}

\author{
T R D Shaw
}

\begin{abstract}
Objective-Assessment of an anterior staircase modification to the standard atrial transseptal technique.

Design-Retrospective report on patients undergoing atrial transseptal puncture. Setting-A regional cardiac centre. Patients-A series of 658 patients catheterised between 1975 and 1991.

Results-Needle entry to the left atrium was obtained in $647(98 \cdot 3 \%)$ patients with needle and catheter entry to the left atrial cavity in 637 patients $(96.8 \%)$. Sustained atrial arrhythmia occurred in six patients $(0.9 \%)$ and pronounced bradycardia in one. Cardiac perforation occurred in three patients $(0 \cdot 5 \%)$ but did not cause cardiac tamponade. There were no deaths or embolic complications caused by the transseptal puncture. The anterior staircase manoeuvre was particularly useful in patients with problem septathat is, when the septum bulged into the right atrial cavity, lacked a detectable limbic edge, was tough or tender, or when the right atrium was considerably enlarged.

Conclusion-The anterior staircase technique is a useful modification to the atrial transseptal puncture technique because it allows repeated passage down the atrial septum without the need for guidewire and needle exchanges for repositioning in the superior vena cava.
\end{abstract}

(Br Heart f 1994;71:297-301)

In the original descriptions of the transseptal technique, ${ }^{1-4}$ the curved needle, after placement within the right atrium, was advanced upwards to the foramen ovale. In 1965, Bloomfield and Sinclair-Smith described a modification of the technique whereby the Brockenbrough catheter was positioned, over

Department of Cardiology, Western General Hospital, Edinburgh

T R D Shaw

Correspondence to: DrTR D Shaw Department of Cardiology, Western General Hospital, Edinburgh EH4 2XU.

Accepted for publication 19 October 1993 the foramen ovale. ${ }^{5}$ This modification has come to be included in the most quoted description of the transseptal technique. Although this method is satisfactory for patients with normal or near normal atrial anatomy, in many patients distortion of the cardiac anatomy make it preferable to undertake several passages down the atrial septum. With the Bloomfield and Sinclair-Smith modification, this requires repeated exchanges of the guidewire and needle each time the catheter is repositioned in the superior vena cava. We describe an anterior staircase method that makes repeated movements down the atrial septum easier.

\section{Methods}

THE ANTERIOR STAIRCASE MANOEUVRE AND TRANSSEPTAL PUNCTURE

After a Brockenbrough or Mullins transseptal catheter had been advanced into the lower half of the right atrium through a $\mathrm{J}$ shaped guidewire, the guidewire was removed and the curved tip Brockenbrough needle was advanced so that its tip lay just within the catheter. The needle was rotated so that the direction arrow at its hub faced 12 o'clock as viewed from below (fig 1). With the needle and catheter constantly moved from side to side between the 10 and 2 o'clock positions, the catheter and needle were advanced to the top of the right atrium and then rotated posteromedially (fig 1). The continual side to side movement as the catheter and needle advanced to the top of the right atrium immediately detected any tendency to snag on the atrial wall. Snagging rarely occurred because when the inferior vena cava enters at the posterior aspect of right atrium there is an anterior space in which this anterior staircase movement can take place (fig 2). When the catheter was rotated posteromedially it was allowed to take up a position between $30^{\circ}$ and $90^{\circ}$ to the horizontal depending on the size of the left atrium ${ }^{7}$ with the greater angulation used in patients with mitral stenosis in whom the left atrium was enlarged and lay behind the right atrium. Patients with only aortic valve disease tended to have a foramen ovale situated more cranially and to require less angulation. If passage down the atrial septum did not show the abrupt posterior movement of the catheter and needle as they crossed the limbic edge or if they were displaced by a distorted or bulging septum, the movement down the septum could be readily repeated by rotating the catheter and needle to the 12 o'clock position and, again with continual 
Figure 1 (1) After the catheter and needle have been placed in the lower half of the right atrium, the curve is rotated to 12 o'clock (as seen from below) and then rotated between $10 o^{\prime}$ clock and 2 $o^{\prime}$ clock as the catheter and needle are advanced to the top of the right atrium. (2) The catheter and needle are rotated posteromedially and drawn down the atrial septum.

(3) If they reach the bottom of the atrial septum without a clear movement into the foramen ovale then the movement down the septum is repeated. (4) The catheter and needle are again rotated to 12 o'clock and moved from side to side while being readvanced to the top of the right atrium.
1
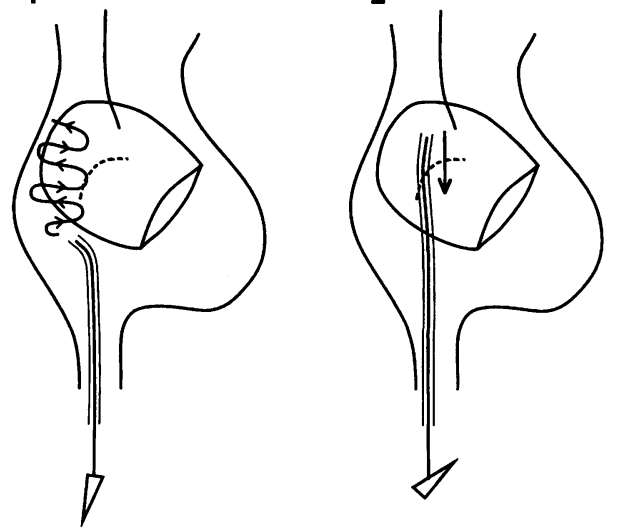

sideways movements, readvanced to the top of the right atrium (fig 1).

When the catheter and needle were seen or felt to have dropped over the limbic edge into the foramen ovale, the catheter was given a slight upward pressure: if the foramen ovale was patent the catheter could then enter the left atrial cavity without exposing the tip of the needle. If the foramen was not probe patent the catheter was kept stationary with the right hand as the needle tip was advanced. The catheter was never allowed to advance until the tip of the needle was confirmed to be within the left atrial cavity by withdrawal of oxygenated blood and by recording a left atrial pressure trace through the needle. If further confirmation was required, contrast was injected into the needle to produce the typical faint swirl within the left atrial cavity. After the catheter was advanced into the left atrial cavity, it was allowed to rotate slightly anticlockwise to help avoid the posterior left atrial wall.

If no movement over the limbic edge was noted then puncture was attempted in the centre of the area of overlap of the right and left atria as judged from fluoroscopy or by
3
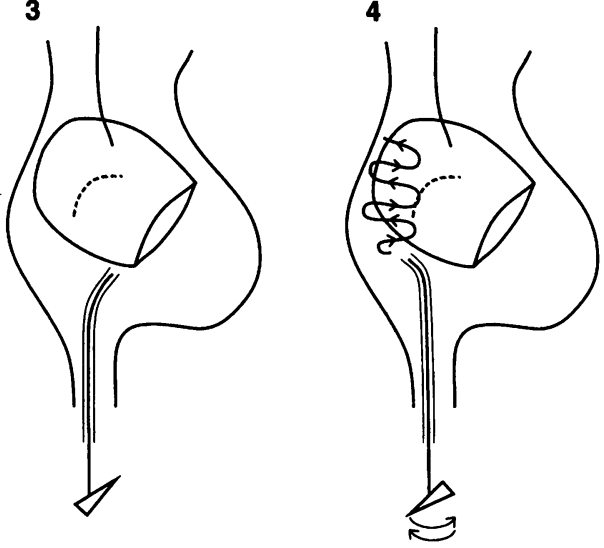

right atrial contrast injection with followthrough until the left atrial cavity was seen. ${ }^{8}$ The modifications of technique used when a problem septum was encountered are described in the results.

Warfarin treatment was normally stopped two days before catheterisation with prothrombin time checked just before the procedure. If heparin was used, it was given after the transseptal puncture had been completed.

All transseptal punctures were carried out with single plane fluoroscopy in the anteroposterior position. Image contrast was adjusted to optimise identification of the left atrial target area. In later procedures the right and left atrial overlap area was routinely identified by right atrial contrast injection with video recording until the left atrium was seen, as described by Inoue. ${ }^{8}$ The target area of overlap was then memorised in relation to the vertebral bodies as seen on fluoroscopy.

Atrial transseptal puncture was not carried out if echocardiography showed thrombus adjacent to the atrial septum, if there had been a recent (within three months) embolic episode, or if atrial myxoma was present. Patients were not excluded because of

Figure 2 Lateral view of right atrial cavity $(R A)$ after contrast injection to show the anterior space used in the anterior staircase manoeuvre: the Brockenbrough catheter and needle (arrows) have been rotated to point anteriorly for side to side advancement to the top of the right atrium (fig 1 ).

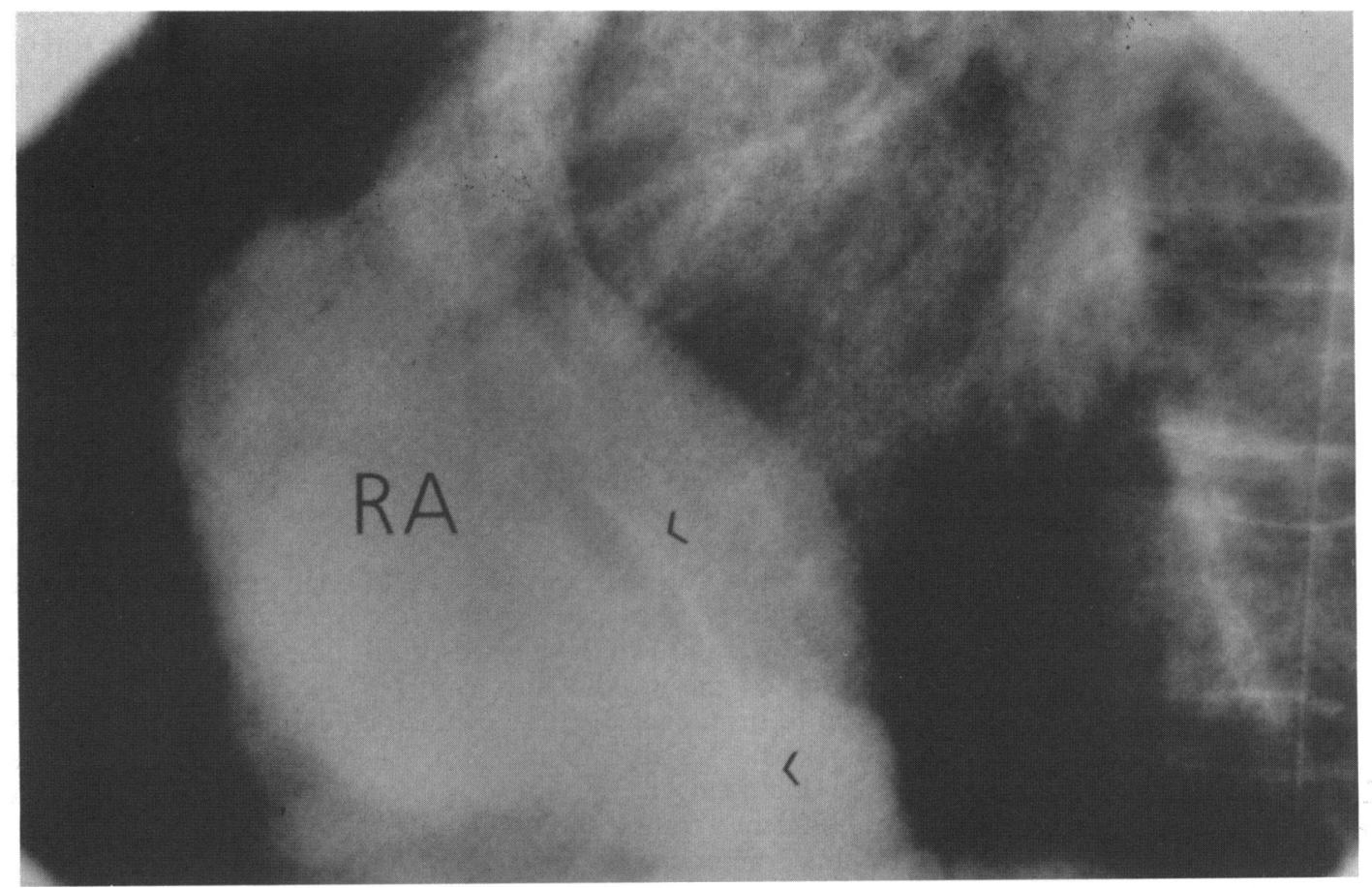


conditions known to make atrial transseptal puncture more difficult-for example, kyphoscoliosis, aneurysm or dilatation of the ascending aorta, displacement of the heart due to lung disease, or need to use the left femoral vein.

\section{PATIENTS}

The anterior staircase technique was used for atrial transseptal puncture in 658 (443 women (67\%) and 215 men (33\%)) patients between 1975 and 1991. In the earlier years, transseptal puncture was attempted in al patients undergoing cardiac catheterisation for assessment of mitral or aortic valve disease but during the past 10 years, transseptal puncture was used only in selected cases when echocardiography had not provided a firm diagnosis. Mitral valve disease was the predominant lesion in $236(36 \%)$ and aortic valve disease in $167(25 \%)$. Eighty patients had combined aortic and mitral valve abnormality, and 11 patients had hypertrophic cardiomyopathy. Miscellaneous indications were present in 14 patients. The series also included 150 patients (23\%) who had atrial septal puncture for balloon dilatation of the mitral valve.

\section{Results}

CROSSING OF ATRIAL SEPTUM

Of the 658 patients in whom atrial transseptal puncture was attempted, the needle entered the left atrial cavity in $647(98 \cdot 3 \%)$. In the other 11 cases, failure was due to a resistant septum in three, inability to aspirate oxygenated blood and record left atrial pressure after repeated attempted puncture in five, and to excessive discomfort of the patient in three.

In an additional 10 cases the needle entered the left atrium but an adult or paediatric Brockenbrough catheter could not be made to follow across the septum. Both needle and catheter crossed the atrial septum in 637 patients $(96 \cdot 8 \%)$

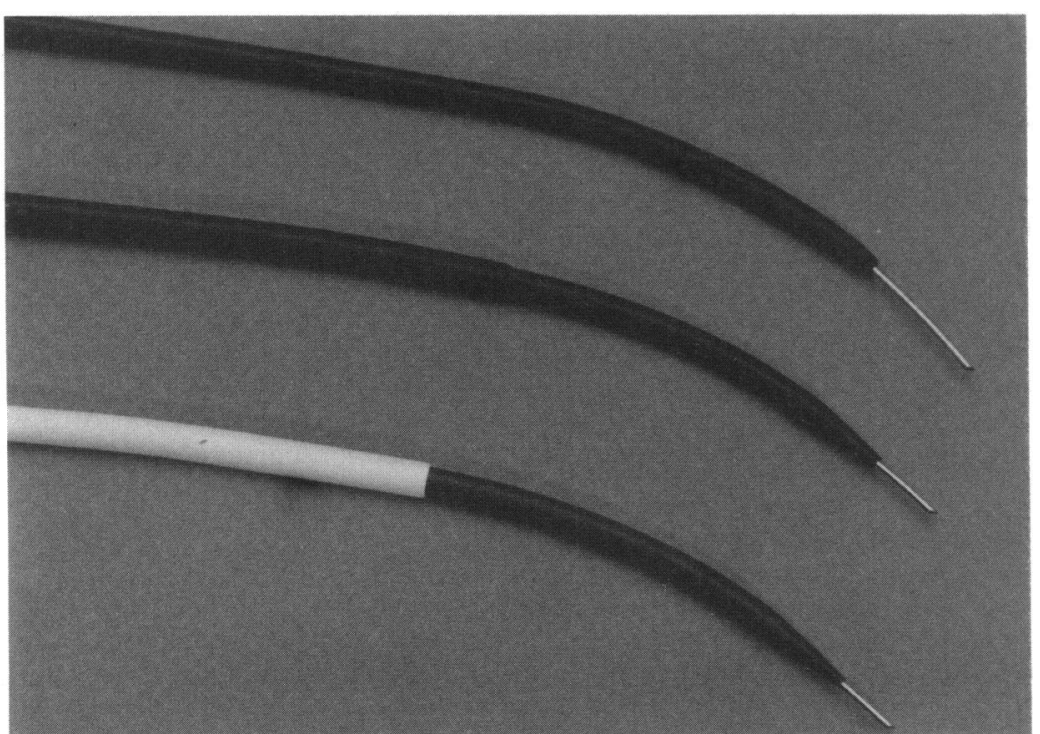

Figure 3 Brockenbrough needle inserted into the adult Brockenbrough catheter (top), the adult Mullins catheter and sheath (middle), and the paediatric Mullins catheter and sheath (bottom)
PROBLEM SEPTA

The anterior staircase manoeuvre was useful when a problem septum was encountered:

\section{Bulging septum}

A tense and bulging left atrium may protrude so far into the right atrial cavity that the catheter and needle are displaced sideways whenever passage down the atrial septum is attempted. Successful crossing could usually be obtained by stopping the downward movement just below the centre of the atrial septum (as identified by fluoroscopy or right atrial contrast injection) and, when the catheter had slid sideways, by then torquing back to bring the catheter and needle tip towards the left atrial target area. A position other than the apex of the bulging septum but within the left atrial target area sometimes had to be accepted. The torque needed in this circumstance could be such that the hub direction arrow no longer exactly corresponded to the needle curve, which had to be judged visually. When in a likely position within the target area, the needle alone was advanced forward. When the needle entered the left atrium, the situation was more stable and the catheter usually followed readily into the left atrial cavity. As several movements down the septum were often necessary before a satisfactory position was found, the anterior staircase manoeuvre helped to avoid delay from repeated guidewire and needle interchanges.

\section{Featureless septum}

With some atrial septa there was no visual or tactile perception of an abrupt jump into the foramen ovale. Again, repeated passage down the septum was needed before this situation was identified. With this type of septum, an area was selected in the middle of the lower portion of the visual target established by fluoroscopy or right atrial contrast injection and an attempt was made to find a snag point on the septum by slight upward movement of the posteromedially orientated catheter and needle when they reached this area during downward movement.

\section{Tough septum}

Some atrial septa, particularly those in patients who have had cardiac surgery, felt tough and resisted crossing by the needle or catheter. In some cases, a stronger than usual push achieved successful crossing but the operator has to be alert to restricting the forward motion as soon as the catheter is within the left atrium. Extremes of force have to be avoided as the needle tip can fracture from its shaft. ${ }^{910}$ If the needle did not cross the septum a different position, usually a lower one within the left atrial visual target area, was tried. If the needle crossed a tough septum but the catheter would not follow then a change from an adult to a paediatric Brockenbrough catheter and needle could be successful. The Mullins (USCI) catheter, however, has a more tapered tip than the Brockenbrough version (fig 3 ) and can cross a 
septum even when the adult and paediatric Brockenbrough catheters have failed. A paediatric Mullins catheter and needle (USCI) are also available.

\section{Tender septum}

Patients often feel a mild discomfort in the throat or chest at the time of atrial septal puncture and should be told that this can be normal. In a few patients discomfort was so intense that they required opiate anaesthesia or, rarely, general anaesthesia. Repeated movements down the septum with the anterior staircase manoeuvre may be tried to seek a less uncomfortable area but often the entire septum shares the tendency to cause discomfort.

\section{Wobbly catheter}

In cases where there was considerable right atrial enlargement, the upward pressure on the catheter and needle for septal puncture could be translated into a sideways movement. This became a greater problem if the septum was tough and resistant. It was helpful to carry out further movements down the septum to seek a lower position for crossing. When the right atrium is very enlarged, Ross suggested manually altering the needle tip into a greater curvature. ${ }^{7}$

\section{COMPLICATIONS}

In six patients $(0.9 \%)$ manipulation of the guidewire or catheter and needle in the right atrium triggered a sustained atrial flutter or atrial fibrillation. This usually occurred at an early stage when the guidewire or catheter entered the right atrium: in no case did the arrhythmia occur during the anterior staircase manoeuvre. One patient had a transient pronounced bradycardia caused by a vasovagal reaction during transseptal puncture.

In one patient $(0 \cdot 2 \%)$ a left haemothorax was detected on return to the ward and a blood transfusion was required. One patient developed a haemopericardium after balloon dilatation of the mitral valve: it was small and did not require aspiration or surgical treatment (two other cases of haemopericardium occurred during balloon dilatation of the mitral valve but were caused by perforation to the left ventricular apex by a balloon catheter and were not related to transseptal puncture). In one patient with a left pleural effusion, the needle and catheter were advanced into the effusion (the aspirate was bright red and a transmitted left atrial pressure waveform had been recorded) but the catheter and needle were withdrawn without any adverse effect.

There were no deaths, detectable embolism, or cardiac tamponade. The Brockenbrough needle never entered the aorta or coronary sinus.

\section{Discussion}

Many patients require more than one passage of the catheter and needle down the atrial septum to achieve atrial puncture. The anterior staircase manoeuvre allowed repeated passage down the septum without the repeated exchange of guidewires and needles that is necessary to re-establish the position within the superior vena cava. The ability to undertake easily several movements down the atrial septum was particularly helpful in those patients with problem septa where there was no clear and immediate drop of the catheter tip over the limbic edge.

The complication rate in this series with the anterior staircase manoeuvre compares favourably with that reported by others. In 1968, Braunwald reported $60(3.4 \%)^{11}$ major complications attributable to transseptal puncture in 1765 patients. Perforation of the heart occurred in 43 patients but led to tamponade in only $21(1.2 \%)$ (the right atrium was perforated in eight of these cases, the left atrium in nine, and the left ventricle in four). Five patients $(0.3 \%)$ had systemic embolism and there were four deaths $(0 \cdot 2 \%)$. This complication rate was comparable with that of other forms of cardiac catheterisation of that era. ${ }^{12}$ Dunn reported only one death in over 1000 transseptal punctures. ${ }^{13} \mathrm{~A}$ low incidence of only minor complications was recorded with modern equipment by Doorey and Goldenberg, ${ }^{14}$ although cardiac tamponade remains a possibility, particularly during interventional transseptal treatments. ${ }^{15}$ When transseptal puncture was used occasionally $(<2 \%)$ for left heart catheterisations from 1978 to 1980 , Shoomaker et al had a mortality of one in $250 .{ }^{16}$

The safety of atrial transseptal puncture is based on never advancing the Brockenbrough or Mullins catheter until the 21 gauge needle tip has been confirmed to be within the left atrial cavity. Although the thin needle tip may be safely withdrawn from a site outside the atria, if the larger catheter shaft perforates the heart there is a risk of cardiac tamponade. If the catheter enters a site other than the atria it should not be withdrawn until the patient is in a location where immediate surgical treatment is available.

Inoue made a valuable contribution to the transseptal technique when he suggested the use of contrast injection to identify the area of overlap of the right and left atrium. ${ }^{8}$ This provides a visual target that supplements the visual and tactile perceptions as the catheter and needle are drawn down towards the foramen ovale, and is particularly helpful when no clear limbic edge is identified. With the introduction of balloon dilatation of the mitral valve, atrial transseptal puncture is now being undertaken by cardiologists previously unfamiliar with the transseptal puncture technique. They will find visualisation of the atria by contrast injection particularly helpful.

Textbook descriptions of atrial transseptal puncture tend to be based on normal or near normal anatomy. Patients with chronic valve disease, however, often have distorted cardiac anatomy that makes repeated passage of the catheter and needle down the atrial septum desirable. In these circumstances the anterior staircase manoeuvre was found to be a useful modification of the atrial transseptal puncture technique. 
1 Ross J. Transseptal left heart catheterisation: a new method of left atrial puncture. Ann Surg 1959; 149:395-401.

2 Ross J, Braunwald E, Morrow AG. Transseptal left atrial puncture: new technique for the measurement of left atrial pressure in man. Am f Cardiol 1959;3 653-5.

3 Brockenbrough EC, Braunwald E. A new technic for left ventricular angiography and transseptal left hear catheterisation. Am $₹$ Cardiol 1960;6:1062-4.

4 Brockenbrough EC, Braunwald E, Ross J. Transseptal left heart catheterisation: a review of 450 studies and description of an improved technic. Circulation 1962; 25:15-21.

5 Bloomfield DA, Sinclair-Smith BC. The limbic edge, a landmark for transseptal left heart catheterisation. landmark for transseptal

6 Baum DS, Grossman W. Transseptal left heart catheterisation. In: Grossman W, Baum DS, eds. Cardiac catheterisation. Angiography and intervention. 4th ed. Philadelphia: Lea and Febiger, 1991:75-9.

7 Ross J. Considerations regarding the technique for transseptal left heart catheterisation. Circulation 1966; 34:391-9.

8 Inoue K. Percutaneous transvenous mitral commissuro- tomy using the Inoue balloon. Eur Heart $f$ 1991; 12(suppl B):99-108.

9 Parker JO, West RO, Fay JE. The Brockenbrough transseptal catheterisation: an unusual complication Circulation 1964;30:743-4.

10 Enghoff E, Cullhed I. Experiences with transseptal left heart catheterisation: a review of 454 studies. Am Heart f 1971;81:398-408.

11 Braunwald E. Transseptal left heart catheterisation Circulation 1968;37(suppl III):74-9.

12 Conti CR, Ross RS. The risks of cardiac catheterisation Am Heart ₹ 1969;78:289-91.

13 Dunn M. Is transseptal catheterisation necessary? f $\mathrm{Am}$ Coll Cardiol 1985;5:1393-4.

14 Doorey AJ, Goldenberg EM. Transseptal catheterisation in adults: an enhanced efficacy and safety by low-volume operators using a "non-standard" technique. ume operators using a "non-standard"

15 Wyman MI, Safian RO, Portway VA, Skillman JO McKay RA, Bain RO. Current complications of diagnostic and therapeutic cardiac catheterisation. $f$ Am Coll Cardiol 1988;12:1400-6.

16 Schoomaker FW, Nampalli KV, Jantz RD. Left atrial and ventricular catheterisation review: losing skills? Cathet Cardiovasc Diagn 1987;13:233-8. 